\title{
Novel symmetries in Weyl-invariant gravity with massive gauge field
}

\author{
K. Abhinav ${ }^{1, \mathrm{a}}$, A. Shukla ${ }^{2, \mathrm{~b}}$, P. K. Panigrahi ${ }^{2, \mathrm{c}}$ \\ ${ }^{1}$ S.N. Bose National Centre for Basic Sciences, JD Block, Sector III, Salt Lake, Kolkata 700106, India \\ ${ }^{2}$ Indian Institute of Science Education and Research Kolkata, Mohanpur 741246, India
}

Received: 14 September 2016 / Accepted: 7 November 2016 / Published online: 22 November 2016

(C) The Author(s) 2016. This article is published with open access at Springerlink.com

\begin{abstract}
The background field method is used to linearize the Weyl-invariant scalar-tensor gravity, coupled with a Stückelberg field. For a generic background metric, this action is found not to be invariant, under both a diffeomorphism and generalized Weyl symmetry, the latter being a combination of gauge and Weyl transformations. Interestingly, the quadratic Lagrangian, emerging from a background of Minkowski metric, respects both transformations independently. The Becchi-Rouet-Stora-Tyutin symmetry of scalar-tensor gravity coupled with a Stuickelberg-like massive gauge particle, possessing a diffeomorphism and generalized Weyl symmetry, reveals that in both cases negativenorm states with unphysical degrees of freedom do exist. We then show that, by combining diffeomorphism and generalized Weyl symmetries, all the ghost states decouple, thereby removing the unphysical redundancies of the theory. During this process, the scalar field does not represent any dynamic mode, yet modifies the usual harmonic gauge condition through non-minimal coupling with gravity.
\end{abstract}

\section{Introduction}

Field theoretic models with scale-invariance have been found to be useful in the context of various cosmological problems $[1,2]$. Scalar-tensor gravity (STG) is one such theory, which possesses local scale-invariance (or Weyl-invariance) and has been widely used in explaining the inflationary properties of the universe [3-5]. It has recently been shown that the conserved current corresponding to the Weyl symmetry of STG, vanishes identically [6]. Thus, although the STG descends from a more general theory of the standard model coupled to gravity [2], at the level of the corresponding Lagrangian, the pure Weyl symmetry has no dynamical role and can be

\footnotetext{
a e-mail: kumar.abhinav@bose.res.in

be-mail: as3756@iiserkol.ac.in

c e-mail: pprasanta@iiserkol.ac.in
}

eliminated by making suitable choice of field variables [7,8]. The physicality of the relevant scalar field can be retrieved by considering the geodesic incompleteness of such theories [9]. We, however, limit ourselves to the STG Lagrangian with no physical mode associated with the Weyl symmetry. In a recent paper, we have shown that there exists a massive scaleinvariant theory, with non-vanishing generalized scale current, when a Stückelberg field is suitably coupled with STG. It is imbibed with a generalized Weyl symmetry: a combination of gauge and local scale symmetry [10]. Remarkably, this STG-Stükelberg theory, with gravity non-minimally coupled to the scalar field, is a consistent theory only when the Weyl and gauge transformations are inter-related, making STG a part of the bigger theory. This aspect may find physical implication in scale-invariant cosmology. As is well known, gravitational theories are notorious to quantize, lacking gaugefixing with respect to the diffeomorphism redundancy. Therefore, the classical analysis first needs to be corroborated with a proper gauge-fixing, for establishing the consistency of the still-extended STG-Stückelberg theory.

Quantization of gravity has been a challenge due to its highly non-linear nature. For this purpose, several important methods e.g., tetrad formalism and background field method, have been developed $[11,12]$. Despite its limitations due to the inherent simplicity, the background field approach has widely been exploited in various gravitational models [13-15]. The fact that the background fields obey classical equations of motion is utilized to attain the lowest order, tree-level quantum action to be quadratic in nature, yielding equations of motion, linear in field variables. For the cases of pure gravity [12], as well as gravity minimally coupled to a dilaton scalar field variable [13], such equations were obtained by identifying proper gauge-fixing against the inherent diffeomorphism redundancy of such systems under generic infinitesimal coordinate transformation. Recently, canonical quantization of massive conformal gravity has been performed in the weak quantum field limit, reveal- 
ing that the quantum massive gravity is unitary and renormalizable, but has ghost states [14]. Furthermore, using the same background field expansion, a generic Weyl-invariant gravitational model with dilatations, in presence of a gauge field, has also been quantized through suitable definition of renormalization group flows [15]. The latter case, including the STG suitably coupled with Abelian gauge field, is of importance in understanding light-matter interaction during conformal phases of the universe (e.g., inflation) and in higher dimensional gravity models with compactification (e.g., Kaluza-Klein gravity and several string theory models). However, even in these background field models, exact gauge-fixing of the conformal dilation gravity, coupled to gauge field, has not been derived so far. Further, consistent separation of the unphysical states, by introduction of proper ghost terms, remains undone till now.

In the present work, we consistently gauge-fix the STGStückelberg theory [10], depicting conformal gravity coupled to massive gauge particle, with the Stückelberg scalar field identified with the scaling scalar of STG, in the background field method. The equations of motion, obtained from a quadratic tree-level 'quantum' Lagrangian, are linear in field variables. Suitable choice of gauge-fixing yields corresponding dynamics with positive semi-definite norm. We adopt the celebrated Becchi-Rouet-Stora-Tyutin (BRST) method, where unitarity and gauge-invariance co-exist [1619], to separate-out negative-norm states, leaving the rest of the dynamic fields 'physical'. This is the first instance of BRST gauge-fixing of such a theory to the best of our knowledge. Though this method allows for construction of quantum brackets at the tree (Lagrangian) level, it is generally accepted [11] that this further ensures consistent quantization up to all orders of perturbation. However, presently we leave the proof of the same for later work, and we limit ourselves to the treelevel dynamics, with physical states identified. To this end, the corresponding Faddeev-Popov ghosts [20] are identified, with invariance under two independent supersymmetric transformations, BRST and anti-BRST [21-24], which are both nilpotent and anticommuting. This enables us to identify the effective physical degrees of freedom of the theory, represented by a graviton and a massive gauge particle, with no dynamics for the scalar field. It is observed that this can be consistent only when Weyl and gauge symmetries are interrelated with a diffeomorphism in a Minkowski background.

The paper is organized as follows. In Sect. 2, we apply the weak field method, with a generic metric as a background and linearize the generalized Weyl-invariant STG-Stückelberg Lagrangian. Further, we show that quadratic Lagrangian is neither invariant under the usual diffeomorphism nor under the generalized Weyl transformation. Remarkably, on taking the background metric to be Minkowskian, the quadratic Lagrangian regains both symmetries, which is depicted in Sect. 3. Subsequently, Sect. 4 is devoted to the discussion of
(anti-)BRST symmetries of the quadratic Lagrangian, emerging from the STG-Stückelberg theory. We show that, for the removal of negative-norm states, one has to combine both diffeomorphism and generalized Weyl transformations, in an appropriate manner. The harmonic gauge-fixing condition gets modified to a specific form of the well-known de Donder gauge due to the non-minimal coupling of gravity with the scalar field. We conclude after summarizing the obtained result and point out future directions of work.

\section{Background field expansion}

The high non-linearity of Einstein gravity prevents its dynamical quantization. A full non-perturbative approach has given rise to the $S L(2, \mathbb{R})$ gravity [11], described through the tetrad formalism [25]. However, high mathematical complexity has prevented the same from yielding practical solutions. Perturbative approaches are relatively simpler, which are based on the physical assumption of smallness of quantum fluctuations against classical curvature. This is very much plausible in a macroscopic universe, such as the present one. The background field approach [13] is applied to expand the complete metric in powers of quantum fluctuations $h_{\mu \nu}$ [12], with the corresponding dynamics governed by their second order contributions, as the first order terms vanish since the classical background fields are always taken to be on-shell [13]. As the corresponding Lagrangian is second order, the equations of motion are linear in $h_{\mu \nu}$, leading to linearized gravity [12].

Quantization of gravity requires, in general, the removal of the inherent redundancy arising from the symmetry under a diffeomorphism transformation,

$$
\begin{aligned}
\delta_{\xi} A_{\mu \nu \cdots}^{\alpha \beta \cdots}= & \xi^{\rho} \partial_{\rho} A_{\mu \nu \cdots}^{\alpha \beta \cdots}+\xi^{\alpha} \partial_{\rho} A_{\mu \nu \cdots}^{\rho \beta \cdots}+\xi^{\beta} \partial_{\rho} A_{\mu \nu \cdots}^{\alpha \rho \cdots}+\cdots \\
& -\xi^{\rho} \partial_{\mu} A_{\rho \nu \cdots}^{\alpha \beta \cdots}-\xi^{\rho} \partial_{\nu} A_{\mu \rho \cdots}^{\alpha \beta \cdots}
\end{aligned}
$$

This makes gravity a gauge theory, with a Fock space containing negative-norm (ghost) states, needing proper gaugefixing. It is a difficult task in this highly non-perturbative theory [11]. In linearized gravity, the obvious approximation of the $S L(2, \mathbb{R})$ diffeomorphism transformation does reduces to one that respects the symmetry of the second order Lagrangian; but only in the case of pure gravity [12]. In general, the presence of other fields coupled to gravity that also transform under a diffeomorphism does not allow it to be the symmetry of the full theory. This makes the theory noncovariant over general coordinate transformation. Therefore, very restricted boundary conditions are required $[14,15]$ to quantize a linear gravitational model with generic matter coupling, while preserving covariance over diffeomorphism.

As a primary need for quantization, by separating physical degrees of freedom from the unphysical ones, we proceed to gauge-fix the combined STG-Stückelberg theory [10], 


$$
\begin{aligned}
\mathcal{L}_{\mathrm{STSt}}= & \sqrt{-\mathcal{G}}\left[\frac{1}{12 \kappa} \mathcal{R} \Phi^{2}+\frac{1}{2 \kappa} \partial_{\mu} \Phi \partial_{\nu} \Phi \mathcal{G}^{\mu \nu}\right. \\
& \left.-\frac{1}{4} \mathcal{F}_{\mu \alpha} \mathcal{F}_{\nu \beta} \mathcal{G}^{\mu \nu} \mathcal{G}^{\alpha \beta}+\frac{m^{2}}{2} \mathcal{G}^{\mu \nu} \mathcal{D}_{\mu} \Phi \mathcal{D}_{\nu} \Phi\right],
\end{aligned}
$$

depicting a generalized Weyl-invariant massive gauge field in presence of curvature. Here, $\kappa=(16 \pi G)$ is a constant of dimension inverse of mass squared, with $G$ being Newtonian gravitational constant, and $R$ is the Ricci scalar. Such a model can be useful in explaining the behavior of a conformal, scale-invariant universe having short-range gauge interactions. Here, the scalar field $\Phi$ couples non-minimally to gravity, defined by the metric $\mathcal{G}_{\mu \nu}$, in the first term of the above Lagrangian. As explained above, quantization of the above theory is much more difficult than Einstein-Hilbert gravity, given the nature of different couplings, including that of the Abelian gauge field $A_{\mu}$ represented by the corresponding field tensor $\mathcal{F}_{\mu \nu}$. Further, it should be noted that $\mathcal{D}_{\mu}=\partial_{\mu}-A_{\mu}$ is a non- $U$ (1) covariant derivative corresponding to the gauge connection [10]. We will opt for a quadratic form of this action, following the background field expansions:

$\mathcal{G}_{\mu \nu}=g_{\mu \nu}+h_{\mu \nu}=g_{\mu \rho}\left(\delta_{\nu}^{\rho}+h_{\nu}^{\rho}\right)$,

$\mathcal{G}^{\mu \nu}=g^{\mu \nu}-h^{\mu \nu}+h_{\rho}^{\mu} h^{\rho v}$,

$h^{\mu \nu}=h^{\nu \mu}, \quad \mathcal{G}_{\mu \nu} \mathcal{G}^{\nu \rho}=\delta_{\mu}^{\rho}=g_{\mu \nu} g^{\nu \rho} ;$

$A_{\mu}=a_{\mu}+b_{\mu} ; \quad \Phi=\varphi+\phi$.

The first terms in the above are classical fields, obeying classical equations of motion (EOM) [10],

$$
\begin{aligned}
\nabla_{\mu} F^{\mu \nu} & =-m^{2} \varphi^{2} a^{\nu}+m^{2} \varphi \partial^{\nu} \varphi, \\
\square \varphi= & \frac{1}{1+\kappa m^{2}}\left[\frac{1}{6} R+\kappa m^{2}\left(\nabla_{\mu} a_{\nu}+a_{\mu} a_{\nu}\right) g^{\mu \nu}\right] \varphi, \\
\mathcal{T}_{\mu \nu}= & \frac{1}{\kappa}\left[\frac{1}{12} G_{\mu \nu} \varphi^{2}+\frac{1}{2} \partial_{\mu} \varphi \partial_{\nu} \varphi-\frac{1}{4} g_{\mu \nu} g^{\alpha \beta} \partial_{\alpha} \varphi \partial_{\beta} \varphi\right. \\
& \left.+\frac{1}{12}\left(g_{\mu \nu} \square-\nabla_{\mu} \nabla_{\nu}\right) \varphi^{2}\right]-\frac{1}{2} F_{\mu}^{\alpha} F_{\nu \alpha} \\
& +\frac{m^{2}}{2} \varphi^{2} \bar{a}_{\mu} \bar{a}_{\nu}-\frac{1}{4} g_{\mu \nu}\left(-\frac{1}{2} F_{\alpha \beta} F^{\alpha \beta}\right. \\
& \left.+m^{2} \varphi^{2} \bar{a}_{\alpha} \bar{a}^{\alpha}\right)=0 ;
\end{aligned}
$$

with

$$
\begin{aligned}
& \square \varphi=\frac{1}{\sqrt{-g}} \partial_{\mu}\left[\sqrt{-g} g^{\mu \nu} \partial_{\nu} \varphi\right], \quad \bar{a}_{\mu}=a_{\mu}-\partial_{\mu} \log \varphi, \\
& G_{\mu \nu}=R_{\mu \nu}-\frac{1}{2} g_{\mu \nu} R .
\end{aligned}
$$

The second terms in Eq. (3) are quantum fluctuations, which are orders of magnitude suppressed. Here, we will follow the treatment by 't Hooft and Veltman [13], carried out for gravity minimally coupled with a scalar field, to obtain the most general second order quantum contribution to the treelevel Lagrangian of Eq. (2).
The most non-trivial term of the action, in view of background field expansion, is the first one, with gravity nonminimally coupled to the scalar field, which will be treated in detail. The background (classical) metric $g_{\mu \nu}$ raises and lowers indices and non-trivially contributes to the background covariant derivative $D_{\mu}$. The gravitational Jacobian expands as [13],

$\sqrt{-\mathcal{G}}=\sqrt{-g}\left(1+\frac{1}{2} h-\frac{1}{4} h^{\mu \nu} h_{\mu \nu}+\frac{1}{8} h^{2}\right) ; \quad h=h_{\mu}^{\mu}$,

where we have used the second expression from the first equation of Eq. (3). Then the first term in Eq. (2) can be expanded, in the powers of the 'quantum' metric $h_{\mu \nu}$ and scalar field $\phi$, in the form,

$\mathcal{L}_{g}:=\frac{1}{12 \kappa} \sqrt{-\mathcal{G}} \mathcal{R} \Phi^{2} \equiv \mathcal{L}_{0}+\mathcal{L}_{1}+\mathcal{L}_{2}+\mathcal{O}(3)$,

wherein $\mathcal{L}_{0}$ has no 'quantum dynamics'. $\mathcal{L}_{1}$ is first order in $h_{\mu \nu}$ and $\phi$, and its contribution is exactly canceled by that coming from the first order contribution from the rest of the Lagrangian, following the classical equations of motion [13]. Hence, the lowest order quantum dynamics arises from $\mathcal{L}_{2}$, which is quadratic in quantum fields, and can be decomposed into the three parts $\mathcal{L}_{2}=\mathcal{L}_{a}+\mathcal{L}_{b}+\mathcal{L}_{c}$, with the expressions

$$
\begin{aligned}
\mathcal{L}_{a}= & \frac{\sqrt{-g}}{12 \kappa}{ }^{2} R \phi^{2}, \\
\mathcal{L}_{b}= & \frac{\sqrt{-g}}{6 \kappa}\left(h_{; \mu \nu}^{\mu \nu}-\square h+h^{\mu \nu 0} R_{\mu \nu}-\frac{1}{2} h^{0} R\right) \varphi \phi, \\
\mathcal{L}_{c}= & \frac{\sqrt{-g}}{12 \kappa}\left[\frac{1}{8}\left(h^{2}-2 h^{\mu \nu} h_{\mu \nu}\right){ }^{0} R-h^{\alpha \mu} h_{\mu \nu}{ }^{0} R_{\alpha}^{\nu}\right. \\
& +\frac{1}{2} h h^{\mu \nu}{ }^{0} R_{\mu \nu}+\frac{1}{4} h^{\mu \nu} \square h_{\mu \nu}-\frac{1}{4} h \square h+\frac{1}{2} h h_{; \mu \nu}^{\mu \nu} \\
& \left.+\frac{1}{2} h^{\mu \nu ; \alpha} h_{\mu \alpha ; \nu}\right] \varphi^{2} .
\end{aligned}
$$

The last expression is obtained through integration by parts and removing total derivatives, with commutator for covariant derivatives being taken into account. Here, the semi-colon indicates covariant derivative with respect to the background metric. Moreover, ${ }^{0} R_{\mu \nu}$ and ${ }^{0} R$ represent the Ricci tensor and scalar with respect to the background metric, respectively.

Expanding the rest of the Lagrangian in Eq. (2), we get

$$
\begin{aligned}
\mathcal{L}_{\mathrm{STSt}}^{(2)} \equiv & \mathcal{L}_{2}+\frac{\sqrt{-g}}{2 \kappa}\left[\frac{1}{8}\left(h^{2}-2 h^{\mu v} h_{\mu \nu}\right) \varphi_{, \alpha} \varphi^{, \alpha}\right. \\
& +\frac{h}{2}\left(2 \varphi_{, \mu} \phi^{, \mu}-h^{\mu v} \varphi_{, \mu} \varphi_{, \nu}\right)+\varphi_{, \mu} h_{\alpha}^{\mu} h^{\alpha \nu} \varphi_{, \nu} \\
& \left.-2 h^{\mu v} \varphi_{, \mu} \phi_{, \nu}+\phi_{, \mu} \phi^{, \mu}\right]-\frac{\sqrt{-g}}{4}\left[\frac { 1 } { 8 } \left(h^{2}\right.\right. \\
& \left.-2 h^{\mu \nu} h_{\mu \nu}\right) A^{\alpha \beta} A_{\alpha \beta}+h\left(A_{\mu \nu} B^{\mu \nu}\right.
\end{aligned}
$$




$$
\begin{aligned}
& \left.-h^{\mu v} A_{\mu}^{\alpha} A_{\nu \alpha}\right)+2 A_{\mu \alpha} h_{\beta}^{\mu} h^{\beta \nu} A_{\nu}^{\alpha} \\
& \left.+h^{\mu v} h^{\alpha \beta} A_{\mu \alpha} A_{\nu \beta}+B_{\mu \nu} B^{\mu \nu}-2 h^{\mu v} A_{\mu}^{\alpha} B_{\nu \alpha}\right] \\
& +\frac{\sqrt{-g}}{2} m^{2}\left[\frac{1}{8}\left(h^{2}-2 h^{\mu v} h_{\mu \nu}\right) d_{\alpha} \varphi d^{\alpha} \varphi+d_{\mu} \phi d^{\mu} \phi\right. \\
& -\frac{1}{2} h h^{\mu \nu} d_{\mu} \varphi d_{\nu} \varphi+d_{\mu} \varphi h^{\mu} h^{\alpha \nu} d_{\nu} \varphi-2 b_{\mu} \varphi d^{\mu} \phi \\
& -b_{\mu} \phi d^{\mu} \varphi+\left(h g^{\mu \nu}-2 h^{\mu \nu}\right)\left(d_{\mu} \varphi d_{\nu} \phi-b_{\mu} \varphi d_{\nu} \varphi\right) \\
& \left.+b_{\mu} b^{\mu} \varphi^{2}\right]+\mathcal{O}\left(h^{3}\right) \\
& =\mathcal{L}_{2}+\mathcal{L}_{\phi}+\mathcal{L}_{\text {gauge }}+\mathcal{L}_{\text {int. }} .
\end{aligned}
$$

This action is quadratic in quantum fields and represents their dynamics. Here, $A_{\mu \nu}$ and $B_{\mu \nu}$ correspond to the background and quantum gauge field strengths, respectively, and $d_{\mu}:=$ $\partial_{\mu}-a_{\mu}$.

The present theory, being coupled to gravity, is expected to be invariant under infinitesimal general coordinate transformation of the form,

$x^{\mu} \rightarrow x^{\mu}+\xi^{\mu}$,

with $\xi^{\mu}$ being small. The corresponding variation of the fields is a diffeomorphism, under which background fields do not change, whereas the quantum fields do [12-14] as [12,13],

$h_{\mu \nu} \rightarrow h_{\mu \nu}+\xi_{(\mu ; v)}+\xi_{;(\mu}^{\alpha} h_{v) \alpha}+\xi^{\alpha} h_{\mu v ; \alpha}$,

$h^{\mu \nu} \rightarrow h^{\mu \nu}-\xi^{(\mu ; \nu)}-\xi^{\alpha ;(\mu} h_{\alpha}^{\nu)}-\xi^{\alpha} h_{; \alpha}^{\mu \nu}$,

$h \rightarrow h+2 \xi_{; \mu}^{\mu}+2 h^{\mu \nu} \xi_{\mu ; \nu}+\xi^{\mu} h_{; \mu}$,

$b_{\mu} \rightarrow b_{\mu}+\xi^{\rho} \partial_{\rho} A_{\mu}+A_{\rho} \partial_{\mu} \xi^{\rho}$,

$\phi \rightarrow \phi+\xi^{\rho} \partial_{\rho} \Phi$.

Here $\xi^{\mu}$ is identified as the diffeomorphism parameter. As observed earlier [13], if smallness of the quantum fields are not taken into account, i.e. the Lagrangian being considered up to all orders of expansion, the transformations in Eq. (11) represent symmetry of the full Lagrangian, not that of the quadratic part. Since the present goal is concerned with the second order contribution, yielding linear EOMs, one needs to reduce these transformations suitably up to certain orders. The obvious choice is to restrict the R.H.S. of Eq. (11) to zero order in quantum fields, as $\xi_{\mu}$ is small [12]. However, unlike pure gravity, the presence of other interacting dynamic fields in the present case forbids such a reduced symmetry of the second order Lagrangian in Eq. (9).

The same is true for the independent generalized Weyl transformations [10], which can be attributed completely to the quantum fields [14] as,

$h_{\mu \nu} \rightarrow h_{\mu \nu}-2 \theta \mathcal{G}_{\mu \nu}, \quad b_{\mu} \rightarrow b_{\mu}+\partial_{\mu} \theta, \quad \phi \rightarrow \phi+\theta \Phi$,

which is again a symmetry of the full Lagrangian in Eq. (2). For the second order Lagrangian, this is no more a symme- try, even when approximated to zero order in quantum fields. This imposes strict restrictions on the background fields, in order to retain both diffeomorphism and generalized Weyl symmetries, in reduced forms, in the Lagrangian. In the following, we obtain such a second order Lagrangian with specific background (classical) fields.

\section{Second order theory with constant background}

Following the case of pure gravity [12] and that of conformal scalar-tensor gravity [14], wherein a reduced diffeomorphism is obtainable in the second order theory for a constant (Minkowski) background metric, for the present case, we consider all three background fields to be constant to yield,

$\mathcal{G}_{\mu \nu}=\eta_{\mu \nu}+h_{\mu \nu}, \quad \Phi=\varphi+\phi, \quad A_{\mu}=b_{\mu}$,

$\sqrt{-} g=1+\frac{1}{2} h-\frac{1}{4} h^{\mu \nu} h_{\mu \nu}+\frac{1}{8} h^{2}+\cdots$,

where $\eta_{\mu \nu}$ is the Minkowski metric and $a_{\mu}=0$. The latter, more restrictive choice owes to the fact that a constant nonzero, non-dynamic vector field destroys the isotropy of the background space, when coupled to the dynamic fields. Further, the generalized Weyl symmetry is broken in the presence of a vector that does not transform. These approximations simplify the second order Lagrangian further as,

$$
\begin{aligned}
\mathcal{L}_{\mathrm{STSt}}^{(2) \mathrm{red}}= & \frac{1}{12 \kappa}\left[\frac{1}{4} h_{\mu \nu} \square h^{\mu \nu}+\frac{1}{2} h \partial_{\mu} \partial_{\nu} h^{\mu \nu}+\frac{1}{2} \partial_{\alpha} h^{\mu \alpha} \partial^{\beta} h_{\mu \beta}\right. \\
& \left.-\frac{1}{4} h \square h\right] \varphi^{2}+\frac{1}{6 \kappa}\left[\partial_{\mu} \partial_{\nu} h^{\mu \nu}-\square h\right] \varphi \phi \\
& +\frac{1}{2 \kappa} \partial_{\mu} \phi \partial^{\mu} \phi-\frac{1}{4} B_{\mu \nu} B^{\mu \nu}+\frac{1}{2} m^{2}\left[\partial_{\mu} \phi \partial^{\mu} \phi\right. \\
& \left.-2 b_{\mu} \varphi \partial^{\mu} \phi+b_{\mu} b^{\mu} \varphi^{2}\right] .
\end{aligned}
$$

Under the reduced diffeomorphism transformations,

$\delta_{\xi} h_{\mu \nu}=\partial_{\mu} \xi_{\nu}+\partial_{\nu} \xi_{\mu}, \quad \delta_{\xi} b_{\mu}=0, \quad \delta_{\xi} \phi=0$,

with quantum fields $b_{\mu}$ and $\phi$ unchanged, the simplified second order Lagrangian is invariant, as it changes by a total derivative term,

$$
\begin{aligned}
\delta_{\xi} \mathcal{L}_{\text {STSt }}^{(2) \text { red }}=\partial_{\mu}[ & \frac{1}{24 \kappa}\left\{\left(\partial_{\nu} \xi_{\alpha}\right) \partial^{\mu} h^{\nu \alpha}-\partial^{\mu}\left(\partial_{\nu} \xi_{\alpha}\right) h^{\nu \alpha}\right. \\
& -\left(\partial_{\alpha} \xi^{\alpha}\right) \partial^{\mu} h+\partial^{\mu}\left(\partial_{\alpha} \xi^{\alpha}\right) h \\
& \left.\left.+2\left(\partial_{\alpha} \xi^{\alpha}\right) \partial_{\nu} h^{\mu \nu}\right\} \varphi^{2}\right] .
\end{aligned}
$$

The generalized Weyl transformation for the quantum fields reduces to,

$\delta_{\theta} h_{\mu \nu}=-2 \theta \eta_{\mu \nu}, \quad \delta_{\theta} b_{\mu}=\partial_{\mu} \theta, \quad \delta_{\theta} \phi=\theta \varphi$, 
which also leaves the Lagrangian in Eq. (14) invariant modulo a total derivative,

$$
\begin{aligned}
\delta_{\theta} \mathcal{L}_{\text {STSt }}^{(2) \text { red }}=\partial_{\mu}[ & \frac{1}{24 \kappa}\left(h \partial^{\mu} \theta-\partial^{\mu} h \theta-4 \theta \partial_{\nu} h^{\mu \nu}\right) \varphi^{2} \\
& \left.+\frac{1}{\kappa} \varphi \phi \partial^{\mu} \theta\right] .
\end{aligned}
$$

As both a diffeomorphism and generalized Weyl transformations are independent, they can be combined as,

$\delta h_{\mu \nu}=\partial_{\mu} \xi_{\nu}+\partial_{\nu} \xi_{\mu}-2 \eta_{\mu \nu} \theta$

$\delta b_{\mu}=\partial_{\mu} \theta, \quad \delta \phi=\theta \varphi$,

with $\delta=\left(\delta_{\xi}+\delta_{\theta}\right)$, leaving the corresponding Lagrangian invariant modulo a total derivative term,

$$
\begin{aligned}
\delta \mathcal{L}_{\mathrm{STSt}}^{(2) \mathrm{red}}= & \partial_{\mu}\left[\frac { 1 } { 2 4 \kappa } \left\{\left(\partial_{\nu} \xi_{\alpha}\right) \partial^{\mu} h^{\nu \alpha}-\partial^{\mu}\left(\partial_{\nu} \xi_{\alpha}\right) h^{\nu \alpha}\right.\right. \\
& \left.-\left(\partial_{\alpha} \xi^{\alpha}\right) \partial^{\mu} h+\partial^{\mu}\left(\partial_{\alpha} \xi^{\alpha}\right) h+2\left(\partial_{\alpha} \xi^{\alpha}\right) \partial_{\nu} h^{\mu \nu}\right\} \varphi^{2} \\
& +\frac{1}{24 \kappa}\left(h \partial^{\mu} \theta-\partial^{\mu} h \theta-4 \theta \partial_{\nu} h^{\mu \nu}\right) \varphi^{2} \\
& \left.+\frac{1}{\kappa} \varphi \phi \partial^{\mu} \theta\right] .
\end{aligned}
$$

As the Lagrangian in (14) is invariant, independently under approximated diffeomorphism and generalized Weyl transformations, both of which represent constraints, the physical degrees of freedom of this system is less than the number of field components. Direct quantization of such a system will lead to a Fock space with negative-norm (energy) states, corresponding to unphysical degrees of freedom. This calls for gauge-fixing each of the two symmetries, so that the equations of motion corresponding to each field correspond only to positive energy states. To this end, the proper gauge-fixing conditions are to be obtained from the respective EulerLagrange equations for fields $h_{\mu \nu}, b_{\mu}$, and $\phi$, which are,

$$
\begin{aligned}
& \square h^{\mu \nu}+\eta^{\mu \nu} \partial_{\alpha} \partial_{\beta} h^{\alpha \beta}-\eta^{\mu \nu} \square h+\partial^{\mu} \partial^{\nu} h+\partial_{\alpha} \partial^{\mu} h^{\alpha \nu} \\
& \quad+\partial_{\alpha} \partial^{v} h^{\alpha \mu}=\frac{4}{\varphi}\left(\eta^{\mu \nu} \square \phi-\partial^{\mu} \partial^{v} \phi\right), \\
& \frac{1}{6 \kappa}\left(\partial_{\mu} \partial_{\nu} h^{\mu \nu}-\square h\right) \varphi=\left(\frac{1}{\kappa}+m^{2}\right) \square \phi-m^{2} \varphi \partial_{\mu} b^{\mu}, \\
& \partial_{\mu} F^{\mu \nu}=m^{2}\left(\varphi \partial^{v} \phi-\varphi^{2} b^{v}\right) .
\end{aligned}
$$

In the gravity sector, the extended harmonic gauge choice [13],

$\partial_{\mu} h^{\mu v}-\frac{1}{2} \partial^{v} h-\frac{2}{\varphi} \partial^{v} \phi=0$,

leads to the EOM for $h_{\mu \nu}$,

$$
\square \bar{H}^{\mu \nu}=0 ; \quad \bar{H}^{\mu \nu}:=h^{\mu \nu}-\frac{1}{2} \eta^{\mu \nu} h-\frac{2}{\varphi} \eta^{\mu \nu} \phi .
$$

Thus, the 'gravitational' mode is represented by a linear combination of quantum metric and scalar fields, which is expected from the non-minimal coupling between the two $[7,8,13]$. This physically makes sense, as the scalar mode corresponds to both scaling and Stückelberg mechanism [10]. Hence, it should not represent any physical degree of freedom. This assertion will be clearer in the following and also in the next section, when (anti-) BRST symmetry will be obtained.

In the vector gauge sector, the independent covariant gauge choice of,

$\partial_{\mu} b^{\mu}+m^{2} \varphi \phi=0$,

much like the Stückelberg case [26], leads to the EOM for $b_{\mu}$,

$$
\left(\square+m^{2} \varphi^{2}\right) b^{v}=0 .
$$

This particular gauge choice is considered due to the fact that the gauge field couples to the scalar one, as in Eq. (2), just like the Stuickelberg theory, with the coupling terms re-arranged. This further justifies the acquired mass,

$m_{b}=m \varphi$,

of the gauge field. Further, on using the gauge-fixing conditions in Eqs. (22) and (24), and the quantum metric EOM of Eq. (23) thereby substituting $h_{\mu \nu}, h$, and $b_{\mu}$, the EOM for $\phi$ reduces to,

$$
\left(\square+m^{2} \varphi^{2}\right) \phi=0,
$$

with a 'mass term',

$m_{\phi}=m \varphi \equiv m_{b}$

The last result is of importance, as it ensures that Eq. (27) can be obtained from Eq. (25) subjected to the gauge-fixing condition of Eq. (24). However, the reverse is not true. This further shows that $\phi$ does not represent an independent dynamical mode, as asserted earlier. The two gauge-fixing conditions relate the scalar field independently to the tensor and vector fields. Also, both these gauge-fixing conditions are required to obtain Eq. (27), and it can be written purely in terms of other two fields, which is not the case for Eqs. (23) and (25). Another way to validate this point is that the Lagrangian in Eq. (2) was obtained through a generalized Weyl scaling of a Proca action in the presence of gravity [10], with the scalar component $\Phi$ exactly being the scaling field [6] and also the Stückelberg-type field. In both regards, it is expected that the scalar component will not yield any dynamics.

Therefore, we have obtained a massless gravitational and massive vector field at the tree-level, subjected to the gaugefixing conditions in Eqs. (22) and (24). This is in accordance to the earlier observations $[7,8,13]$, with modification coming due to the additional gauge coupling [10]. As symmetric 
$h_{\mu \nu}$ has 10 independent components and $b_{\mu}$ has 4 ; 4 modified harmonic constraints Eq. (22), 4 coming from the generic coordinate transformation Eq. (10) [12] and 1 vector gauge constraint Eq. (24) leave the net physical degrees of freedom of the system to be $(10+4)-(4+4+1)=5$, with 2 for graviton and 3 for massive gauge particle, which is expected physically. This aspect will be further elucidated, while introducing the ghost fields for the theory, in the next section. As the exact gauge-fixing conditions have been achieved, by obtaining 'physical' equations of motion (23) and (25) with positive spectra, the (anti-)BRST symmetries of the present system can be obtained, which is demonstrated in the next section.

\section{4 (Anti-)BRST transformations}

Our goal is to identify the physical degrees of freedom of the present theory, which has redundancies arising from both the diffeomorphism and generalized Weyl transformations. To this end, we opt for the BRST approach, wherein auxiliary gauge-fixing conditions are introduced at the expense of introducing Faddeev-Popov ghost and anti-ghost fields, representing negative-norm states arising from the gauge redundancies of the original theory [20]. Effectively, this increase in variables re-locates the over-counting of degrees of freedom from the gauge to the (anti-)ghost sector, as gauge transformation is replaced by the corresponding (anti-)BRST transformations [16-19]. The latter is nilpotent and as it is done off-shell, one is left with only physical degrees of freedom in the gauge sector, defined in a Fock space with positive semi-definite norm.

In the present case, the diffeomorphism gauge sector contains only one vector ghost term in the Lagrangian, since it is confined only to $h_{\mu \nu}$, with $\phi$ being non-dynamic. Similarly, the generalized Weyl redundancy will lead to one scalar ghost term, which affects all the three fields, including $b_{\mu}$ and $\phi$. This is because the gauge-fixing term for $h_{\mu \nu}$ remains invariant under the corresponding nilpotent (anti-)BRST transformations. Finally, the combined diffeomorphism and generalized Weyl gauge sector is dealt with, achieving the physical states of the full theory, through necessary inclusion of both vector and scalar (anti-)ghost fields.

\subsection{For diffeomorphism symmetry}

Here, we consider the (anti-)BRST symmetry of the Lagrangian with respect to the diffeomorphism. This is exclusive to the gravitational sector, through introduction of corresponding vector (anti-)ghost fields, made possible by its independence from the generalized Weyl symmetry in the present theory. For this purpose, we gauge-fix only the gravitational sector of the complete Lagrangian in Eq. (14), in the modified har- monic gauge of Eq. (22), and introduce corresponding vector (anti-)ghost field $\left(\bar{c}_{\mu}\right) c_{\mu}$, with ghost number $(-1) 1$, to yield,

$$
\begin{aligned}
\mathcal{L}_{\mathrm{GF} 1}^{(2)}= & \frac{1}{12 \kappa}\left[\frac{1}{4} h_{\mu \nu} \square h^{\mu \nu}+\frac{1}{2} h \partial_{\mu} \partial_{\nu} h^{\mu \nu}+\frac{1}{2} \partial_{\alpha} h^{\mu \alpha} \partial^{\beta} h_{\mu \beta}\right. \\
& \left.-\frac{1}{4} h \square h\right] \varphi^{2}+\frac{1}{6 \kappa}\left[\partial_{\mu} \partial_{\nu} h^{\mu \nu}-\square h\right] \varphi \phi+\frac{1}{2 \kappa} \partial_{\mu} \phi \partial^{\mu} \phi \\
& -\frac{1}{4} B_{\mu \nu} B^{\mu \nu}+\frac{1}{2} m^{2}\left[\partial_{\mu} \phi \partial^{\mu} \phi-2 b_{\mu} \varphi \partial^{\mu} \phi+b_{\mu} b^{\mu} \varphi^{2}\right] \\
& -\frac{1}{24 \kappa}\left(\partial_{\nu} h^{\mu \nu}-\frac{1}{2} \partial^{\mu} h-2 \varphi^{-1} \partial^{\mu} \phi\right)^{2}+\frac{i}{12 \kappa} \partial^{\mu} \bar{c}^{\nu} \partial_{\mu} c_{\nu} .
\end{aligned}
$$

Here, and afterwards, we explicitly use the Feynman-' $t$ Hooft gauge. In the above Lagrangian, the gauge-fixing term can be linearized by adding the Nakanishi-Lautrup type auxiliary vector field $B_{\mu}$ as,

$$
\begin{aligned}
\mathcal{L}_{\mathrm{GF} 1}^{(2)} \equiv & \frac{1}{12 \kappa}\left[\frac{1}{4} h_{\mu \nu} \square h^{\mu \nu}+\frac{1}{2} h \partial_{\mu} \partial_{\nu} h^{\mu \nu}+\frac{1}{2} \partial_{\alpha} h^{\mu \alpha} \partial^{\beta} h_{\mu \beta}\right. \\
& \left.-\frac{1}{4} h \square h\right] \varphi^{2}+\frac{1}{6 \kappa}\left[\partial_{\mu} \partial_{\nu} h^{\mu \nu}-\square h\right] \varphi \phi \\
& +\frac{1}{2 \kappa} \partial_{\mu} \phi \partial^{\mu} \phi-\frac{1}{4} B_{\mu \nu} B^{\mu \nu}+\frac{1}{2} m^{2}\left[\partial_{\mu} \phi \partial^{\mu} \phi\right. \\
& \left.-2 b_{\mu} \varphi \partial^{\mu} \phi+b_{\mu} b^{\mu} \varphi^{2}\right]+\frac{1}{24 \kappa} B_{\mu} B^{\mu} \\
& +\frac{1}{12 \kappa} B_{\mu}\left(\partial_{\nu} h^{\mu \nu}-\frac{1}{2} \partial^{\mu} h-2 \varphi^{-1} \partial^{\mu} \phi\right)+\frac{i}{12 \kappa} \partial^{\mu} \bar{c}^{\nu} \partial_{\mu} c_{\nu},
\end{aligned}
$$

with $B_{\mu}=-\left(\partial_{\nu} h^{\mu \nu}-\frac{1}{2} \partial^{\mu} h-2 \varphi^{-1} \partial^{\mu} \phi\right)$ on-shell. The action corresponding to the above Lagrangian is invariant under the following off-shell nilpotent (anti-)BRST transformations:

$s_{b}^{(1)} h_{\mu \nu}=\partial_{\mu} c_{\nu}+\partial_{\nu} c_{\mu}, \quad s_{b}^{(1)} c_{\mu}=0, \quad s_{b}^{(1)} \bar{c}_{\mu}=i B_{\mu}$,

$s_{b}^{(1)} B_{\mu}=0, \quad s_{b}^{(1)} b_{\mu}=0, \quad s_{b}^{(1)} \phi=0$,

$s_{a b}^{(1)} h_{\mu \nu}=\partial_{\mu} \bar{c}_{\nu}+\partial_{\nu} \bar{c}_{\mu}, \quad s_{a b}^{(1)} \bar{c}_{\mu}=0, \quad s_{a b}^{(1)} c_{\mu}=-i B_{\mu}$,

$s_{a b}^{(1)} B_{\mu}=0, \quad s_{a b}^{(1)} b_{\mu}=0, \quad s_{a b}^{(1)} \phi=0$,

as the Lagrangian transforms by total derivative terms, respectively, as

$$
\begin{aligned}
s_{b}^{(1)} \mathcal{L}_{\mathrm{GF} 1}^{(2)}= & \partial_{\mu}\left[\frac { 1 } { 2 4 _ { \kappa } } \left\{\left(\partial_{\nu} c^{\alpha}\right) \partial^{\mu} h^{\nu \alpha}-\partial^{\mu}\left(\partial_{\nu} c_{\alpha}\right) h^{\nu \alpha}\right.\right. \\
& \left.-\left(\partial_{\alpha} c^{\alpha}\right) \partial^{\mu} h+\partial^{\mu}\left(\partial_{\alpha} c^{\alpha}\right) h+2\left(\partial_{\alpha} c^{\alpha}\right) \partial_{\nu} h^{\mu \nu}\right\} \varphi^{2} \\
& \left.+\frac{1}{12 \kappa} B^{\nu} \partial^{\mu} c_{\nu}\right], \\
s_{a b}^{(1)} \mathcal{L}_{\mathrm{GF} 1}^{(2)}= & \partial_{\mu}\left[\frac { 1 } { 2 4 \kappa } \left\{\left(\partial_{\nu} \bar{c}^{\alpha}\right) \partial^{\mu} h^{\nu \alpha}-\partial^{\mu}\left(\partial_{\nu} \bar{c}_{\alpha}\right) h^{\nu \alpha}\right.\right. \\
& \left.-\left(\partial_{\alpha} \bar{c}^{\alpha}\right) \partial^{\mu} h+\partial^{\mu}\left(\partial_{\alpha} \bar{c}^{\alpha}\right) h+2\left(\partial_{\alpha} \bar{c}^{\alpha}\right) \partial_{\nu} h^{\mu \nu}\right\} \varphi^{2} \\
& \left.-\frac{1}{12 \kappa} B^{\nu} \partial^{\mu} \bar{c}_{\nu}\right] .
\end{aligned}
$$

Therefore, the gauge redundancy corresponding to a diffeomorphism is removed by the inclusion of the gauge-fixing 
term, whereas the corresponding symmetry is now compensated by the (anti-)BRST symmetry of the (anti-)ghost fields. The physical credibility of the above procedure is reflected by the fact that, in the original Lagrangian of Eq. (14), the symmetric tensor field $h_{\mu \nu}$ had 10 degrees of freedom, unlike the physical graviton, which has only 2 . To identify the correct number, one has to go to the level of equations of motion (on-shell) to figure out eight constraint equations, four from harmonic gauge and the other four from the remaining choice of the corresponding parameter $\xi_{\mu}$ in coordinate transformation, as discussed in the previous section. On the other hand, introduction of vector (anti-)ghost fields, with four negative degrees of freedom each, corresponds to the same physics at the level of the Lagrangian itself (off-shell). The auxiliary field is always on-shell and does not have any dynamics. This enables $h_{\mu \nu}$ in $\mathcal{L}_{\mathrm{GF} 1}^{(2)}$ to represent exact physical degrees of freedom and thus, with only positive energy states in the Fock space, with the negative energy ones attributed to the (anti-)ghost fields.

It is to be noted that only the gravitational sector is considered above, mainly to demonstrate the physical aspects of the same. Equation (30) represents a two-component tensor field with semi-positive norm, coupled to the fields $b_{\mu}$ having gauge redundancy, and $\phi$. Thus, only the prior can represent tree-level quantum dynamics. To achieve the full spectrum of physical states for $\mathcal{L}_{\text {STSt }}^{(2) \text { red }}$, we need to fix the generalized Weyl redundancy corresponding to the other two fields, which is done later.

\subsection{For generalized Weyl symmetry}

In order to gauge-fix the generalized Weyl symmetry, which includes tensor, vector and scalar sectors of $\mathcal{L}_{\text {STSt }}^{(2) \text { red }}$, unlike the diffeomorphism before, we extend $\mathcal{L}_{\text {STSt }}^{(2) \text { red }}$ to

$$
\begin{aligned}
\mathcal{L}_{\mathrm{GF} 2}^{(2)}= & \frac{1}{12 \kappa}\left[\frac{1}{4} h_{\mu \nu} \square h^{\mu \nu}+\frac{1}{2} h \partial_{\mu} \partial_{\nu} h^{\mu \nu}+\frac{1}{2} \partial_{\alpha} h^{\mu \alpha} \partial^{\beta} h_{\mu \beta}\right. \\
& \left.-\frac{1}{4} h \square h\right] \varphi^{2}+\frac{1}{6 \kappa}\left[\partial_{\mu} \partial_{\nu} h^{\mu \nu}-\square h\right] \varphi \phi+\frac{1}{2 \kappa} \partial_{\mu} \phi \partial^{\mu} \phi \\
& -\frac{1}{4} B_{\mu \nu} B^{\mu \nu}+\frac{1}{2} m^{2}\left[\partial_{\mu} \phi \partial^{\mu} \phi-2 b_{\mu} \varphi \partial^{\mu} \phi+b_{\mu} b^{\mu} \varphi^{2}\right] \\
& +\frac{B^{2}}{2}+B\left(\partial \cdot b+m^{2} \varphi^{2} \phi\right)^{2}+i \partial^{\mu} \bar{c} \partial_{\mu} c+i m^{2} \varphi^{2} \bar{c} c
\end{aligned}
$$

with scalar (anti-)ghost $(\bar{c}) c$ and Nakanishi-Lautrup auxiliary scalar $B$, with $B=-\left(\partial \cdot b+m^{2} \varphi^{2} \phi\right)$ on-shell [27]. The above Lagrangian, under the (anti-)BRST transformations

$$
\begin{aligned}
& s_{b}^{(2)} h_{\mu \nu}=-2 c \eta_{\mu \nu}, \quad s_{b}^{(2)} b_{\mu}=\partial_{\mu} c, \quad s_{b}^{(2)} \phi=\varphi c, \\
& s_{b}^{(2)} c=0, \quad s_{b}^{(2)} \bar{c}=i B, \quad s_{b}^{(2)} B=0, \\
& s_{a b}^{(2)} h_{\mu \nu}=-2 \bar{c} \eta_{\mu \nu}, \quad s_{a b}^{(2)} b_{\mu}=\partial_{\mu} \bar{c}, \quad s_{a b}^{(2)} \phi=\varphi \bar{c}, \\
& s_{a b}^{(2)} \bar{c}=0, \quad s_{a b}^{(2)} c=-i B, \quad s_{a b}^{(2)} B=0,
\end{aligned}
$$

changes by the total derivative terms,

$$
\begin{aligned}
s_{b}^{(2)} \mathcal{L}_{\mathrm{GF} 2}^{(2)}= & \partial_{\mu}\left[\frac{1}{24 \kappa}\left(h \partial^{\mu} c-\partial^{\mu} h c-4 c \partial_{\nu} h^{\mu \nu}\right) \varphi^{2}\right. \\
& \left.+\frac{1}{\kappa} \varphi \phi \partial_{\mu} c+B \partial^{\mu} c\right], \\
s_{a b}^{(2)} \mathcal{L}_{\mathrm{GF} 2}^{(2)}= & \partial_{\mu}\left[\frac{1}{24 \kappa}\left(h \partial^{\mu} \bar{c}-\partial^{\mu} h \bar{c}-4 \bar{c} \partial_{\nu} h^{\mu \nu}\right) \varphi^{2}\right. \\
& \left.+\frac{1}{\kappa} \varphi \phi \partial_{\mu} \bar{c}-B \partial^{\mu} \bar{c}\right],
\end{aligned}
$$

respectively, leaving the corresponding action invariant.

As in the case of diffeomorphism before, only partial physical spectrum has been achieved, as is evident from the corresponding equations of motion, with only Eq. (25) being reproduced, yielding positive energy states only for the vector field $b_{\mu}$. The fact that the gauge-fixing condition in Eq. (24) relates $b_{\mu}$ with the scalar field $\phi$ makes the 2 negative degrees of freedom of the (anti-)ghost scalars compensating together against the redundant combination of 4 positive degrees of freedom of $b_{\mu}$ and 1 of $\phi$. This leaves three physical degrees of freedom for $b_{\mu}$, as $\phi$ does not represent a dynamical mode, analogous to the Stückelberg theory [28], having a positive spectrum and thereby being the only physical field, corresponding to the particular symmetry, coupled to $h_{\mu \nu}$ that contains unphysical states presently. Therefore, it is clear that though a diffeomorphism and generalized Weyl gauge-fixing terms are mutually independent, all the dynamical components (field) of the present theory cannot be made physical, at the same time, by any one of them. Direct evidence of the same is the fact that Eq. (27) for $\phi$ is not obtained in any of these 'independent' procedures, and thus, the scalar degree of freedom remains ill-defined in both of them. This is because, in the second order theory, $\phi$ is the only field, out of the three, that directly couples to the other two. Therefore, the positive-norm Fock space for the full theory can only be achieved with both diffeomorphism and generalized Weyl gauge-fixings applied simultaneously, which is achieved below.

\subsection{For combined diffeomorphism and generalized Weyl symmetry}

As discussed above, in order to obtain the complete physical spectrum of the theory, both gauge-fixing conditions in Eqs. (22) and (24) are to be applied. Including the corresponding auxiliary Nakanishi-Lautrup and (anti-)ghost fields, of both vector and scalar types, the full tree-level quantum Lagrangian can be written as

$$
\begin{aligned}
\mathcal{L}_{\mathrm{GF}}^{(2)}= & \frac{1}{12 \kappa}\left[\frac{1}{4} h_{\mu \nu} \square h^{\mu \nu}+\frac{1}{2} h \partial_{\mu} \partial_{\nu} h^{\mu \nu}+\frac{1}{2} \partial_{\alpha} h^{\mu \alpha} \partial^{\beta} h_{\mu \beta}\right. \\
& \left.-\frac{1}{4} h \square h\right] \varphi^{2}+\frac{1}{6 \kappa}\left[\partial_{\mu} \partial_{\nu} h^{\mu \nu}-\square h\right] \varphi \phi+\frac{1}{2 \kappa} \partial_{\mu} \phi \partial^{\mu} \phi
\end{aligned}
$$




$$
\begin{aligned}
& -\frac{1}{4} B_{\mu \nu} B^{\mu \nu}+\frac{1}{2} m^{2}\left[\partial_{\mu} \phi \partial^{\mu} \phi-2 b_{\mu} \varphi \partial^{\mu} \phi+b_{\mu} b^{\mu} \varphi^{2}\right] \\
& +\frac{1}{24 \kappa} B_{\mu} B^{\mu}+\frac{1}{12 \kappa} B_{\mu}\left(\partial_{\nu} h^{\mu \nu}-\frac{1}{2} \partial^{\mu} h-2 \varphi^{-1} \partial^{\mu} \phi\right) \\
& +\frac{B^{2}}{2}+B\left(\partial \cdot b+m^{2} \varphi^{2} \phi\right)^{2}+\frac{i}{12 \kappa} \partial^{\mu} \bar{c}_{\nu} \partial_{\mu} c^{\nu} \\
& +i \partial^{\mu} \bar{c} \partial_{\mu} c+i m^{2} \varphi^{2} \bar{c} c .
\end{aligned}
$$

Thus, the unphysical ghost sector, imbibing negative-norm states, is completely decoupled from the other dynamical fields, leaving them associated only with positive semidefinite energies, and thereby removing the gauge redundancies due to both diffeomorphism and generalized Weyl symmetries. The latter is now re-structured as the following BRST and anti-BRST transformations:

$$
\begin{aligned}
& s_{b} h_{\mu \nu}=\partial_{\mu} c_{\nu}+\partial_{\nu} c_{\mu}-2 \eta_{\mu \nu} c, \quad s_{b} b_{\mu}=\partial_{\mu} c, \\
& s_{b} \phi=\varphi c, \quad s_{b} \bar{c}_{\mu}=i B_{\mu}, \quad s_{b} \bar{c}=i B, \\
& s_{b}\left[c_{\mu}, \quad c, \quad B_{\mu}, \quad B\right]=0, \quad s_{a b} h_{\mu \nu}=\partial_{\mu} \bar{c}_{\nu}+\partial_{\nu} \bar{c}_{\mu}-2 \eta_{\mu \nu} c, \\
& s_{a b} b_{\mu}=\partial_{\mu} c, \quad s_{a b} \phi=\varphi c, \quad s_{a b} c_{\mu}=-i B_{\mu}, \\
& s_{a b} c=-i B, \quad s_{a b}\left[\bar{c}_{\mu}, \bar{c}, \quad B_{\mu}, B\right]=0 .
\end{aligned}
$$

Under these transformations, the Lagrangian $\mathcal{L}_{\mathrm{GF}}^{(2)}$, respectively, transforms by the total derivatives,

$$
\begin{aligned}
s_{b} \mathcal{L}_{\mathrm{GF}}^{(2)}= & \partial_{\mu}\left[\frac { 1 } { 2 4 \kappa } \left\{\left(\partial_{\nu} c^{\alpha}\right) \partial^{\mu} h^{\nu \alpha}-\partial^{\mu}\left(\partial_{\nu} c_{\alpha}\right) h^{\nu \alpha}\right.\right. \\
& \left.-\left(\partial_{\alpha} c^{\alpha}\right) \partial^{\mu} h+\partial^{\mu}\left(\partial_{\alpha} c^{\alpha}\right) h+2\left(\partial_{\alpha} c^{\alpha}\right) \partial_{\nu} h^{\mu \nu}\right\} \varphi^{2} \\
& +\frac{1}{\kappa} \varphi \phi \partial_{\mu} c+\frac{1}{24 \kappa}\left(h \partial^{\mu} c-\partial^{\mu} h c-4 c \partial_{\nu} h^{\mu \nu}\right) \varphi^{2} \\
& \left.+\frac{1}{12 \kappa} B^{\nu} \partial^{\mu} c_{\nu}+B \partial^{\mu} c\right], \\
s_{a b} \mathcal{L}_{\mathrm{GF}}^{(2)}= & \partial_{\mu}\left[\frac { 1 } { 2 4 \kappa } \left\{\left(\partial_{\nu} \bar{c}^{\alpha}\right) \partial^{\mu} h^{\nu \alpha}-\partial^{\mu}\left(\partial_{\nu} \bar{c}_{\alpha}\right) h^{\nu \alpha}\right.\right. \\
& \left.-\left(\partial_{\alpha} \bar{c}^{\alpha}\right) \partial^{\mu} h+\partial^{\mu}\left(\partial_{\alpha} \bar{c}^{\alpha}\right) h+2\left(\partial_{\alpha} \bar{c}^{\alpha}\right) \partial_{\nu} h^{\mu \nu}\right\} \varphi^{2} \\
& +\frac{1}{\kappa} \varphi \phi \partial_{\mu} \bar{c}+\frac{1}{24 \kappa}\left(h \partial^{\mu} \bar{c}-\partial^{\mu} h \bar{c}-4 \bar{c} \partial_{\nu} h^{\mu \nu}\right) \varphi^{2} \\
& \left.-\frac{1}{12 \kappa} B^{\nu} \partial^{\mu} \bar{c}_{\nu}-B \partial^{\mu} \bar{c}\right],
\end{aligned}
$$

leaving the action invariant. Both relevant fields are now properly gauge-fixed, and they satisfy the 'free' equations (23), (25), and (27), which can lead to quantum effects, due to respective couplings.

Physically, it is crucial to evaluate the actual degrees of freedom of the complete Lagrangian $\mathcal{L}_{\mathrm{GF}}^{(2)}$, in the (anti-)BRST formalism. We count 10 tensor $\left(h_{\mu \nu}\right), 4$ vector $\left(b_{\mu}\right)$ and 1 scalar $(\phi)$ component of dynamical fields, yielding 15 positive degrees of freedom (not considering the on-shell, nondynamic auxiliary fields). The negative degree of freedom count is 10 (4 vector and 1 scalar each for both ghost and antighost kind). Therefore, the system has 5 physical degrees of freedom, 2 for $h_{\mu \nu}$ (graviton) and 3 for $b_{\mu}$ (massive gauge boson). This is the result we obtained in the last section, but at the level of equations of motion. This re-ensures the non-dynamical status of $\phi$, governed by Eq. (27), as in the previous section.

Therefore, the physical domain of the STG-Stuickelberg theory has been completely identified in the weak field approximation. This is done through the (anti-) BRST procedures, ensuring the same to be off-shell, thus enabling EulerLagrange equations derived out of the Lagrangian in Eq. (36) to yield proper tree-level propagators for both $\bar{H}_{\mu \nu}$ and $b_{\mu}$. In the generalized graviton sector, the gauge-fixed EOM reads,

$$
\frac{1}{2}\left[\eta^{\mu \alpha} \eta^{\nu \beta}+\eta^{\mu \beta} \partial^{\nu} \partial^{\alpha}+\eta^{\nu \beta} \partial^{\mu} \partial^{\alpha}-\eta^{\mu \nu} \partial^{\alpha} \partial^{\beta}\right] \bar{H}_{\alpha \beta}=0,
$$

which is exactly of the form for pure gravity under harmonic gauge [12], yielding the corresponding propagator,

$\Delta^{\mu \nu, \alpha \beta}=-\frac{i}{2} \frac{\eta^{\mu \alpha} \eta^{\nu \beta}+\eta^{\mu \beta} \eta^{\nu \alpha}-\eta^{\mu \nu} \eta^{\alpha \beta}}{p^{2}+i \epsilon}$,

representing an effective massless mode [12,13]. Similarly, the gauge propagator is same as the Stuickelberg propagator [28],

$\Delta^{\mu \nu}=-i \frac{\eta^{\mu \nu}}{p^{2}-m_{b}^{2}}$.

The present gauge-fixing of the quadratic Lagrangian, having both a reduced diffeomorphism and generalized Weyl symmetry, is of the most general form and complements the results in $[14,15]$, especially those with canonical approach [14]. This is sufficient to quantize the theory at the treelevel, with bare propagators specified. However, the quantum corrections to a particular field, due to the interaction with another, can provide corrections to the bare structures, and even lead to anomalies. We leave such possibilities for future investigations.

\section{Conclusions}

In conclusion, the STG-Stückelberg theory with non-minimal scalar-gravity coupling, following background field method, led to inconsistencies for a non-trivial background metric; as both the diffeomorphism and generalized Weyl symmetry of the classical theory are not respected. However, very interestingly, in Minkowskian background, this theory can be consistently gauge-fixed by retaining both symmetries, where all the negative-norm states (ghost states) are decoupled from the physical Fock space. Earlier, the quantization of massive conformal gravity has been carried out by showing that the theory is renomrmalizable but has ghost states [14]. Here, the ghost-free Fock space has been 
achieved, after relating all symmetries; namely a diffeomorphism and generalized Weyl symmetry. Further, the graviton and the massive gauge particle represent the entire dynamics, and the scalar field remains non-dynamical in the present case.

Using the well-established BRST technique, the quadratic Lagrangian, emerging from the STG-Stückelberg theory, has been independently gauge-fixed, with respect to the diffeomorphism and generalized Weyl transformations. In the gravitational sector, corresponding to a diffeomorphism only, an appropriately extended harmonic gauge condition, of the de Donder form, is required, yielding a pair of vector (anti-) ghost fields. This leaves-out the negative-norm states with respect to the dynamical vector field $b_{\mu}$ in the theory. On the other hand, gauge-fixing only the extended Weyl gauge redundancy yields three physical degrees of freedom for the gauge field $b_{\mu}$, as the scalar field $\phi$ is not dynamical. Thus, negative-norm states exist in both the cases, and the complete physical sector of the STG-Stückelberg theory is not identified, preventing the corresponding quantization. The identification of the full physical Fock space of the quadratic STG-Stückelberg Lagrangian requires a vector and a scalar pair of (anti-)ghost terms, obtained by combining the corresponding transformations. All the unphysical degrees of freedom of fields $h_{\mu \nu}$ and $b_{\mu}$ have been removed, yielding 5 physical degrees of freedom, 2 for the graviton $\left(h_{\mu \nu}\right)$ and 3 for the massive gauge boson $\left(b_{\mu}\right)$. It will be worthwhile to investigate effective theories emerging from the present one, with interesting possibilities in areas like conformal gravity and gauge theories, as well as in condensed-matter systems. Some of these questions will be investigated in future work.

Open Access This article is distributed under the terms of the Creative Commons Attribution 4.0 International License (http://creativecomm ons.org/licenses/by/4.0/), which permits unrestricted use, distribution, and reproduction in any medium, provided you give appropriate credit to the original author(s) and the source, provide a link to the Creative Commons license, and indicate if changes were made.

Funded by SCOAP ${ }^{3}$.

\section{References}

1. R. Kallosh, A. Linde, J. Cosmol. Astropart. Phys. 07, 2 (2013)

2. I. Bars, P.J. Steinhardt, N. Turok, Phys. Rev. D 89, 061302(R) (2014)

3. W.F. Kao, Phys. Lett. A 154, 1 (1991)

4. M. Artymowski, Y. Ma, X. Zhang, Phys. Rev. D 88, 104010 (2013)

5. C. Csáki, N. Kaloper, J. Serra, J. Terning, Phys. Rev. Lett. 113, $161302(2014)$

6. R. Jackiw, S.-Y. Pi, Phys. Rev. D 91, 067501 (2015)

7. C. Callan, S. Coleman, R. Jackiw, Ann. Phys. (N.Y.) 59, 42 (1970)

8. S. Deser, Ann. Phys. (N.Y.) 59, 248 (1970)

9. I. Bars, A. James, Phys. Rev. D 93, 044029 (2016)

10. A. Shukla, K. Abhinav, P. K. Panigrahi, Class. Quantum Grav. 33, 235008 (2016)

11. N. Nakanishi, I. Ojima, Covariant Operator Formalism of Gauge Theory and Quantum Gravity (World Scientific, Singapore, 1990)

12. S. Carroll, Spacetime and Geometry, An Introduction to General Relativity (Addison Wesley, San Fransisco, 2004)

13. G. 't Hooft, M. Veltman, Ann. Inst. Henn. Poincaré XX, 69 (1974)

14. F.F. Faria, Eur. Phys. J. C 76, 188 (2016)

15. C. Pagani, R. Percacci, Class. Quantum Gravity 31, 115005 (2014)

16. C. Becchi, A. Rouet, R. Stora, Phys. Lett. B 52, 344 (1974)

17. C. Becchi, A. Rouet, R. Stora, Commun. Math. Phys. 42, 127 (1975)

18. C. Becchi, A. Rouet, R. Stora, Ann. Phys. (N.Y.) 98, 287 (1976)

19. I.V. Tyutin, Lebedev Institute Preprint, Report No: FIAN-39 (1975, unpublished)

20. L.D. Faddeev, V.N. Popov, Phys. Lett. B 52, 29 (1967)

21. L. Bonora, P. Pasti, M. Tonin, Nuovo Cimento A 64, 307 (1981)

22. R.P. Malik, Int. J. Mod. Phys. A 22, 3521 (2007)

23. R.P. Malik, Eur. Phys. J. C 55, 687 (2008)

24. R. Kumar, S. Krishna, A. Shukla, R.P. Malik, Int. J. Mod. Phys. A 29, 1450135 (2014)

25. A. Ashtekar, Lectures on Non-perturbative Canonical Gravity (World Scientific, Singapore, 1991)

26. A. Das, Lectures on Quantum Field Theory (World Scientific, Singapore, 2008)

27. A. Shukla, S. Krishna, R.P. Malik, Adv. High Energy Phys. 2015, $258536(2015)$

28. H. Ruegg, M.R. Ruegg, Int. J. Mod. Phys. A 19, 3265 (2004) 